\title{
Postoperative ventricular septal hematoma: Natural history of two pediatric cases
}

\author{
Richard Jensen, MD, ${ }^{a}$ Pamela Burg, MD, ${ }^{a}$ Chris Anderson, MD, ${ }^{a}$ Carl Garabedian, MD, ${ }^{a}$ Hrair Garabedian, MD, ${ }^{a}$
} Leland Siwek, MD, ${ }^{\mathrm{b}}$ and Neil Worrall, MD, ${ }^{\mathrm{b}}$ Spokane, Wash

丹 Supplemental material is available online.

$\mathrm{W}$ e describe the case histories of 2 patients less than 6 months of age in whom ventricular septal hematomas developed in the early postoperative period. Both cases were managed expectantly with complete resolution and no long-term sequelae.

\section{Clinical Summaries}

Patient 1 . The first patient was seen at 2 days of age with poor feeding and a cardiac murmur. An echocardiogram confirmed the diagnosis of type B interrupted aortic arch, posterior malalignment ventricular septal defect (VSD), and ascending aortic hypoplasia. After stabilization, she underwent complete 1-stage surgical repair. With the use of deep hypothermic circulatory arrest, the aortic arch was reconstructed and augmented with pulmonary homograft material. The malaligned VSD was approached through the tricuspid valve. A series of pledget-supported interrupted sutures were placed through the defect margins and a patch of Dacron material secured into position. The patient was weaned from bypass on low-dose dopamine with no rhythm or hemodynamic concerns. Total cardiopulmonary bypass time was 103 minutes. Aortic crossclamp time was 89 minutes. The circulatory arrest time was 43 minutes. A prominent ventricular septal mass with cystic characteristics was noted on postoperative day 1 by transthoracic imaging and persisted on subsequent studies (Figures 1 and Figures e1, $A$ ). The postoperative course was otherwise uneventful and the patient was discharged on the eighth postoperative day. Outpatient follow-up documented resolution of the ventricular septal mass over the course of the ensuing few months (Figure E1, $B$ and $C$ ).

Patient 2. In the second patient, a large membranous VSD associated with significant pulmonary overcirculation was diagnosed at 3 months of age. Surgical intervention was performed at

From the Northwest Center for Congenital Heart Disease ${ }^{\mathrm{a}}$ and Northwest Heart and Lung Associates, ', Sacred Heart Children's Hospital, Spokane, Wash.

Received for publication Dec 21, 2006; accepted for publication Jan 2, 2007.

Address for reprints: Richard Jensen, MD, Northwest Center for Congenital Heart Disease, Sacred Heart Children's Hospital, 101 West Eighth Ave, Suite 4300, Spokane, WA 99220-2555 (E-mail: rjensen@nwcchd.com).

J Thorac Cardiovasc Surg 2007;133:1651-2

$0022-5223 / \$ 32.00$

Copyright () 2007 by The American Association for Thoracic Surgery doi:10.1016/j.jtcvs.2007.01.052

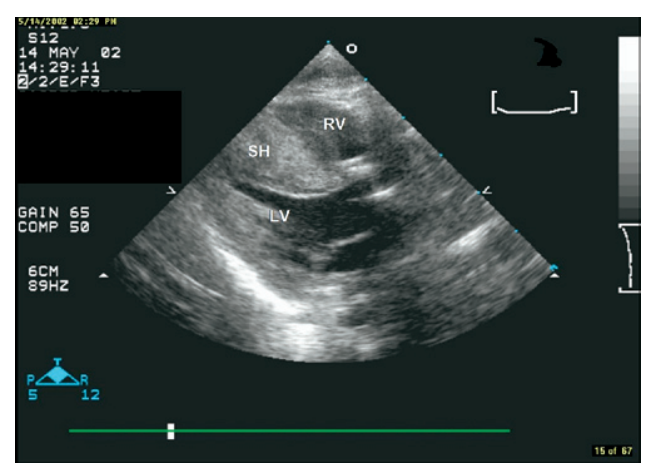

Figure 1. Transthoracic image from patient 1. Early parasternal long-axis view demonstrating septal hematoma. SH, Septal hematoma; $R V$, right ventricle; $L V$, left ventricle.

the age of 3 months. The exposure to the VSD was through the tricuspid valve. The VSD was located in the membranous septum and extended toward the outlet septum. Multiple pledget-supported horizontal mattress sutures were placed around the VSD margin and along the fibrous continuity of the semilunar valves. A Dacron patch was secured into position. The cardiopulmonary bypass time and aortic crossclamp time were 114 and 89 minutes, respectively. An early transesophageal echocardiogram demonstrated the presence of a large fluid-filled space occupying the entire region between the right and left ventricular septae (Figure E2, A). After reversal of heparin, the fluid-filled space organized (Figure 2) and was associated with abnormal septal wall motion, transient global left ventricular dysfunction, and transient second-degree atrioventricular block. Separation from bypass occurred with the aid of low-dose inotropes and temporary epicardial pacing. The postoperative course was complicated by junctional ectopic tachycardia, treated with intravenous amiodarone. Second-degree atrioventricular block and junctional ectopic tachycardia both resolved within the first 12 postoperative hours. The remaining course was uneventful and she was discharged to her home on postoperative day 8. Outpatient follow-up revealed no rhythm abnormalities or clinical symptoms. A follow-up echocardiogram performed 7 weeks after the operation documented resolution of the hematoma and no regional wall motion abnormalities (Figure E2, $B$ and $C$ ).

\section{Discussion}

Ventricular septal hematoma is a rare phenomenon that to our knowledge has not been reported in the setting of a VSD repair. Intramyocardial hematoma has been reported in the setting of acute myocardial infarction, chest wall trauma, aortic valve disease, and coronary artery bypass surgery. ${ }^{1,2}$ Myocardial hematoma 


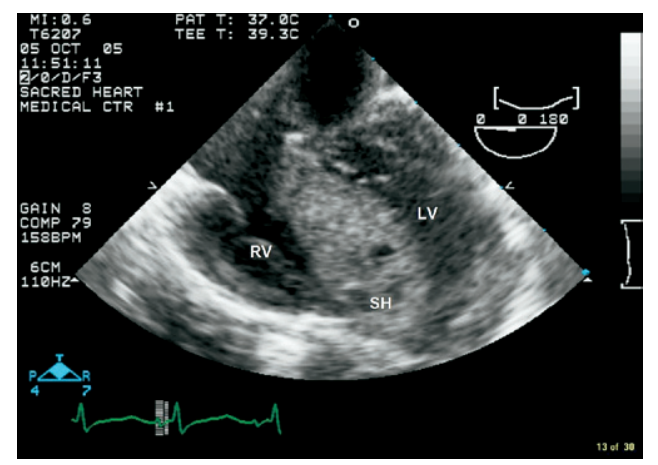

Figure 2. Transesophageal image from patient 2 after bypass. Four-chamber view after heparin reversal reveals hematoma organization. $S H$, Septal hematoma; $R V$, right ventricle; $L V$, left ventricle.

formation may cause hemodynamic or conduction abnormalities and may lead to serious short-term results, including high-grade heart block, outflow obstruction, and tamponade..$^{3,4}$

The interventricular septum is typically considered a homogenenous, simple structure. On the basis of the aforementioned clinical findings and embryologic considerations, there likely is a potential space in all patients that, rarely, can be a site of bleeding and subsequent hematoma formation. Presumably, injury to a septal perforating artery may occur during placement of the septal patch sutures and, during anticoagulation, active bleeding may ensue.
The expectant management of these 2 cases would support a generally benign course for ventricular septal hematomas. Although one of the reported patients did have transient heart block, junctional ectopic tachycardia, and transient ventricular dysfunction, it was not clear that these findings were actually related to the hematoma. Similar rhythm and hemodynamic issues are not uncommon in the early postoperative setting, particularly in small children after heart surgery.

Pediatric cardiologists, sonographers, and cardiothoracic surgeons should be aware of this entity, because perioperative decisions regarding treatment could potentially complicate otherwise straightforward cases. Potential approaches to management include watchful waiting, as in our 2 cases, versus hematoma evacuation. Review of larger congenital heart surgery registries might be helpful and would lend insight into the relative frequency, technical associations, and natural history of this rare postoperative complication.

\section{References}

1. Vargas-Barron J, Romero-Cardenas A, Roldan F, Molina-Carrion M, Avila-Casado C, Villavicencio R, et al. Long-term follow-up of intramyocardial dissecting hematomas complicating acute myocardial infarction. $J$ Am Soc Echocardiogr. 2005;18:1422.el-6.

2. Hutchins GM, Moore GW. Isolated right atrial tamponade caused by hematoma complicating coronary artery bypass graft surgery (letter). Arch Pathol Lab Med. 1980;104:612-4.

3. Pliam MB, Sternlieb JJ. Intramyocardial dissecting hematoma: an unusual form of subacute cardiac rupture. J Card Surg. 1993;49:203-6.

4. Tsubo T, Araki I, Ishihara H, Matsuki A. Atrial septal hematoma immediately after cardiac surgery: detection using transesophageal echocardiography. Anesthesiology. 1995;83:620-1. 

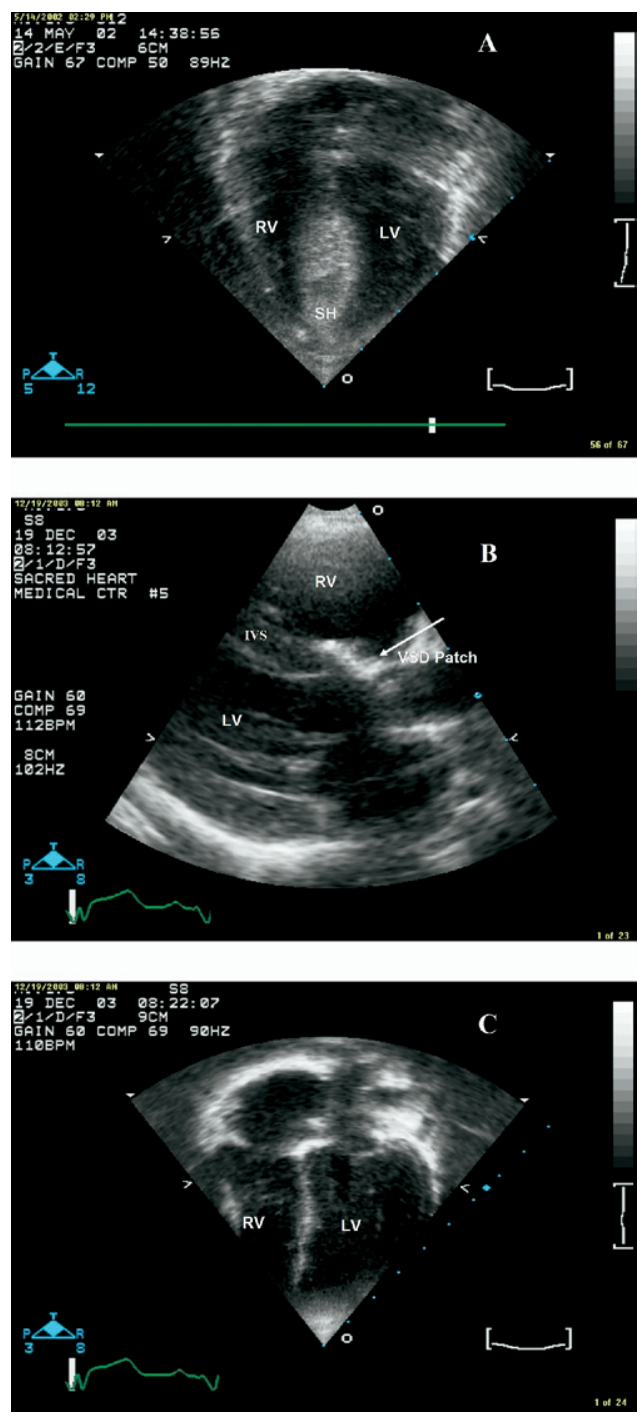

Figure E1. Transthoracic images from patient 1. A, Apical fourchamber view. B, Parasternal long-axis image after resolution of hematoma. C, Apical four-chamber view after hematoma resolution. $S H$, Septal hematoma; $R V$, right ventricle; $L V$, left ventricle; IVS, interventricular septum. Arrow, Ventricular septal defect patch.
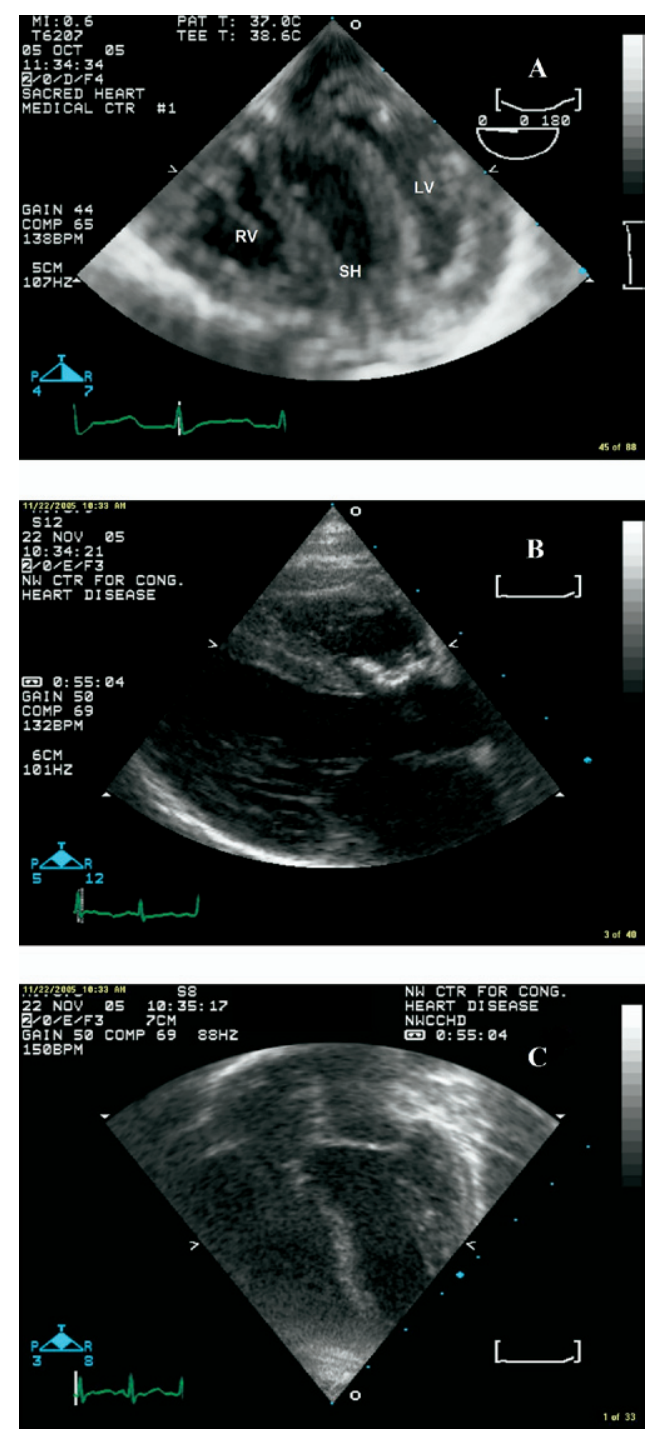

Figure E2. Transthoracic echocardiogram from patient 2 after bypass. A, Four-chamber view demonstrating fluid-filled septal hematoma before heparin reversal. Transthoracic images from patient 2, 6 weeks postoperatively. B, Parasternal long-axis view shows prominent ventricular septal defect patch but normalappearing septum. C, Apical four-chamber view documenting no residual hematoma. $S H$, Septal hematoma; $R V$, right ventricle; $L V$, left ventricle. 